\title{
Measurement of the Parallax of PSR B0950+08 Using the VLBA
}

\author{
Walter F. Brisken ${ }^{1}$, John M. Benson ${ }^{2}$, A. J. Beasley ${ }^{3}$, Edward B. Fomalont ${ }^{3}$, W. M. Goss ${ }^{2}$, \\ S. E. Thorsett ${ }^{4}$
}

\begin{abstract}
A new technique has been developed to remove the ionosphere's distorting effects from low frequency VLBI data. By fitting dispersive and non-dispersive components to the phases of multifrequency data, the ionosphere can be effectively removed from the data without the use of $a$ priori calibration information. This technique, along with the new gating capability of the VLBA correlator, was used to perform accurate astrometry on pulsar B0950+08, resulting in a much improved measurement of this pulsar's proper motion $\left(\mu_{\alpha}=-1.6 \pm 0.4 \mathrm{mas} / \mathrm{yr}, \mu_{\delta}=29.5 \pm 0.5\right.$ mas/yr) and parallax ( $\pi=3.6 \pm 0.3$ mas). This puts the pulsar at a distance of $280 \pm 25$ parsecs, about twice as far as previous estimates, but in good agreement with models of the electron density in the local bubble.
\end{abstract}

Subject headings: astrometry — techniques: interferometric — pulsars: individual (PSR B0950+08)

\section{Introduction}

Accurate radio pulsar distances are important for population and birthrate modeling and, combined with measurements of dispersion and Faraday rotation, for studies of the ionized interstellar medium and Galactic magnetic fields. Parallax measurements can firmly fix the bottom rungs of the pulsar distance ladder, but parallaxes of even the closest pulsars are only a few milliarcseconds in amplitude, and such measurements are available for only nine pulsars (see Toscano et al. 1999 for a recent list). ${ }^{5}$

Four of these parallax measurements were made using timing studies of millisecond pulsars with particularly stable spin periods. The others were made with various VLBI techniques. The use of calibration sources within the primary antenna

\footnotetext{
${ }^{1}$ Dept. of Physics, Princeton University, Princeton, NJ 08544, email: walterfb@nacho.princeton.edu

${ }^{2}$ NRAO, P.O. Box O, Socorro, NM 87801 2475

${ }^{3}$ NRAO, Edgemont Road, Charlottesville, VA 22903-

${ }^{4}$ Dept. of Astr. and Astroph., University of California, Santa Cruz, CA 95064

${ }^{5}$ An optical parallax is also available for the pulsar B0633+17 (Geminga), which has no known radio counterpart.
}

beam shows promise, as demonstrated by Fomalont et al. (1999). Observing at L band with 25 meter telescopes provides a primary beam size of about 30 minutes of arc. Only a small fraction of pulsars have calibrators within this small distance. The number of accessible target pulsars is greatly increased by the use of calibrators at greater offset angles, up to several degrees, but differential ionospheric phase delays make high precision comparison measurements very difficult.

Most pulsars are weak radio sources at high frequencies, where array resolution is high and ionospheric effects are minimized. Observations are often made at $\mathrm{L}$ band (1.4 to $1.7 \mathrm{GHz}$ ) as a compromise, but even at $\mathrm{L}$ band the ionosphere remains a significant obstacle. We have developed a new calibration technique to remove the effects of the ionosphere from the data and used it to measure a revised parallax of PSR B0950+08.

\section{Observations}

At $\mathrm{L}$ band, the VLBA provides simultaneous access to a large spanned bandwidth and is sensitive enough to detect a pulsar within the changing ionosphere's timescale of about one minute. Recently, gating the VLBA correlator became possible. This allows the correlator to be turned off 
during the off pulse phase of the pulsar, increasing the signal to noise of the pulsar by a factor of 3 to 6 , depending on the pulse duty cycle. For PSR B0950+08 the improvement was a factor of 3.6.

We observed the pulsar simultaneously in eight $8 \mathrm{MHz}$ bands ranging from $1410 \mathrm{MHz}$ to 1730 $\mathrm{MHz}$. Each band was split into thirty two 250 $\mathrm{kHz}$ channels. The VLBA antennas nod back and forth between the pulsar and the calibrator with cycle times of about five minutes. Seven epochs from 1994 to 1999 were observed near parallax extrema. The first three epochs were ungated and used $1004+141$ as a calibrator source. Its distance of seven degrees from the pulsar made phase referencing difficult. These data were useful for developing techniques and understanding limitations but were not of high enough quality for accurate astrometry. The more recent epochs used J0946+1017, less than three degrees distant from PSR B0950+08, as a calibrator.

Most astrometry at low frequency is done at night since the ionosphere phase fluctuations are two or three times stronger during the day. Since parallax measurements are most effective when the target and the sun are 90 degrees apart in the sky, these observations need to be done at sunrise and sunset - the times when the ionosphere changes most rapidly.

\section{Data Reduction}

\subsection{Phase-referencing}

This is a VLBI phase-referenced (Taylor et al. 1999) experiment with an additional calibration step. A strong source, J0946+1017, was used as the reference source. Using this object's known position, the fringe rates and delays were measured and interpolated over the pulsar scans. The calibrator is self-calibrated so that its structure can be modeled. The self-calibration phases are interpolated over the pulsar. This simple phase referencing determines the pulsar's position to less than 30 mas. The phase center is shifted to the pulsar's position and the 32 spectral channels within each $8 \mathrm{MHz}$ band are averaged together. The large number of channels prevents bandwidth smearing from decorrelating the long baseline visibilities.

\subsection{The Ionosphere}

The ionosphere, like the free electrons in the ISM, introduces a frequency dependent delay to the radio waves. Unlike for the ISM, this delay can be drastically different above each antenna since the ionosphere has structure on all scales, ranging from meter-sized variations to the bulge caused by the sun's radiation which stretches a third of the way around Earth. The ionosphere is also different along different lines of sight from the same antenna. Since the lines of sight from each telescope to the pulsar and calibrator puncture the ionosphere at different points, phase-referencing only calibrates away the bulk ionosphere over each antenna, leaving spatial gradients in the ionosphere uncorrected. These gradients can change significantly on time scales shorter than one minute and are large enough to move the pulsar by as much as 10 mas per degree of source separation. A phase referenced image with no ionospheric correction is shown in figure 1 .

At a given time on a given baseline, the phasereferenced visibility phases have this structure:

$$
\phi(\nu)=\underbrace{\left[\frac{1}{c}(U \ell+V m)+\delta A\right]}_{\text {non-dispersive }} \nu+\underbrace{\delta B \frac{1}{\nu}}_{\text {dispersive }}
$$

$U$ and $V$ are the projected baseline lengths, $\ell$ and $m$ are the pulsar's vector distance from the phase center (the quantity that this experiment is trying to measure), and $\delta A \nu$ is the phase due to the unmodeled wet troposphere and is very small, introducing less than 45 degrees of phase. These three terms are non-dispersive and hence have the same frequency dependence. $\delta A$ is measured in $\mathrm{rad} / \mathrm{GHz}$, and $\delta B$ is in rad.GHz. $\delta B / \nu$ is the phase due to uncorrected ionosphere. Since the remaining ionosphere is the only dispersive term, it can be separated from the non-dispersive sources of phase.

This separation, however, is complicated by phase ambiguity. For a given set of phase measurements, a whole family of solutions can be formed since $n \cdot 2 \pi$ can be added to the original phases. Because our bandwidth is limited, any integral value of $n$ will generate a different solution with an almost equally good fit. It is fortunate that the value of $\delta A$ is always small enough that a good guess of the pulsar's position constrains the ambiguity to 
a single value of $n$.

\subsection{Determining the Pulsar's Position}

A bootstrapping approach is used to determine the pulsar's position. Using just the 5 Southwestern VLBA antennas ( Los Alamos NM, Pie Town NM, Fort Davis TX, Kitt Peak AZ, and Owens Valley CA ) with a maximum baseline of $1500 \mathrm{~km}$, a separate image is made for each $8 \mathrm{MHz}$ band in the phase-referenced data. Figures 2 and 3 show two such images. Plotting the measured positions as a function of frequency reveals a trend - the points line up in frequency order. If we assume a simplified model of the ionosphere as a single wedge lying above all the antennas then we would expect the pulsar to appear at $\vec{P}(\nu)=\overrightarrow{P_{\infty}}+\vec{w} / \nu^{2}$. Fitting for $\overrightarrow{P_{\infty}}$, the extrapolated infinite frequency position, yields an initial guess of the pulsar's position ( the star on figures 2 and 3 ) with a precision of 3-10 mas. The wedge model is certainly far from correct over the whole Earth but works fairly well for the Southwest array. Because the ionosphere changes so quickly, a 15 to 30 minute snapshot usually works better than imaging all of the data.

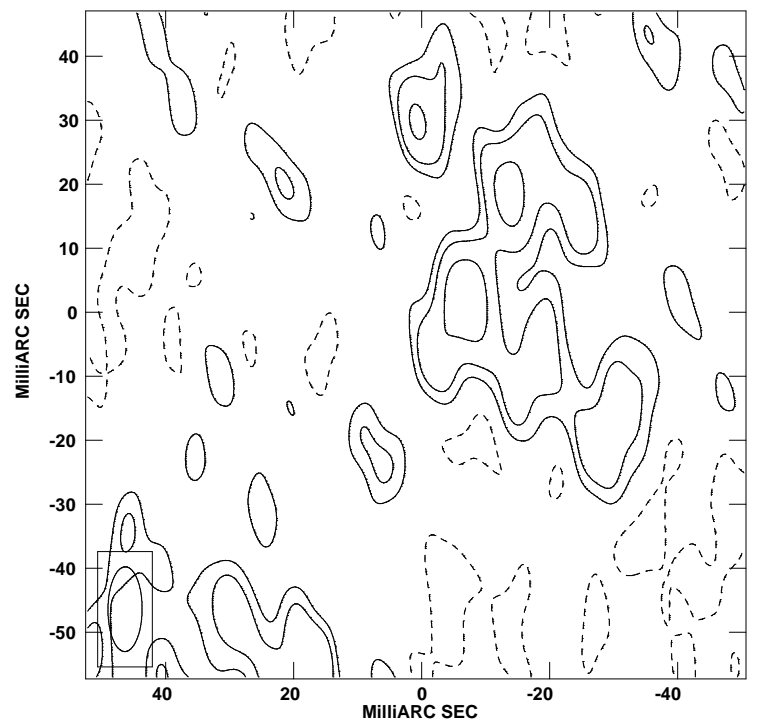

Fig. 1.- Image of pulsar before removing the ionosphere. All of the flux is from the pulsar, scattered by the ionosphere. The beam is 4 by 11 mas. The lowest contour level is at $5 \mathrm{mJy}$ and a factor of 2 separates contours. All data from all telescopes were used to produce this image.

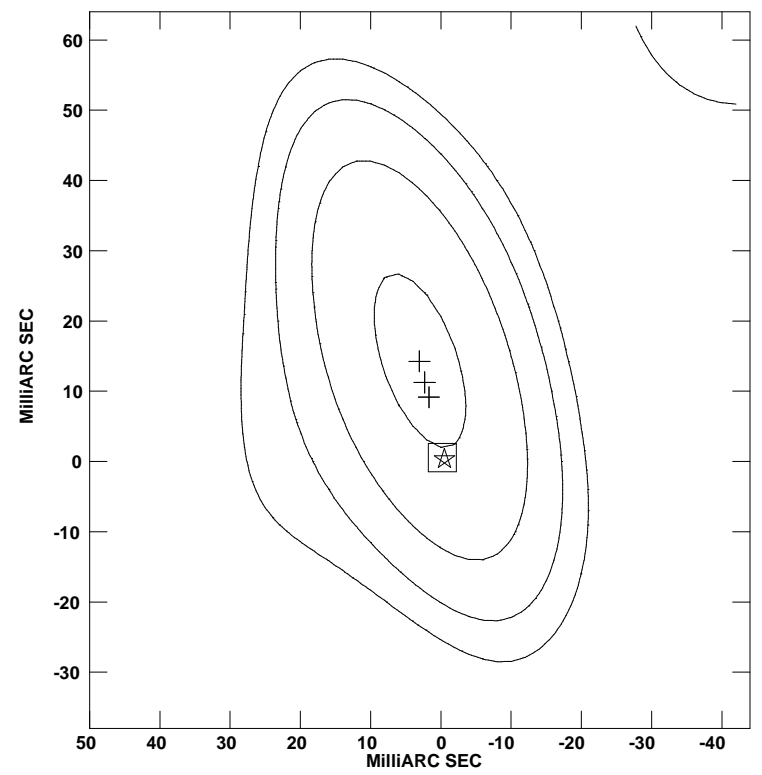

Fig. 2. - 30 minute snapshot made at $1.40 \mathrm{GHz}$ using only the 5 Southwestern antennas. The beam size and shape match that of the secondhighest contour. The three pluses designate measured location of the pulsar at three different frequencies; from the top, these frequencies are: 1.40 $\mathrm{GHz}, 1.63 \mathrm{GHz}$, and $1.73 \mathrm{GHz}$. The star indicates the inferred true location of the pulsar by extrapolating the measured positions to infinite frequency. The center of the square is where the pulsar was determined to be using the techniques shown in this paper.

To get antenna-based phases as a function of frequency, the pulsar is self-calibrated with a solution interval of between twelve and thirty seconds and the antenna-based phase corrections are recorded. The self-calibration phases cannot be applied to the pulsar, as that would move the pulsar to the phase center. For each of the Southwestern antennas, the nondispersive and dispersive components of the self-calibration solutions are fit, holding the geometric $\ell$ and $m$ terms fixed. This is reasonable since we know the pulsar's location to much less than 30 mas, the fringe spacing of the longest Southwestern baseline. The other antennas are initially ignored as the phase contribution due to geometric delay is significant on the longer baselines. A new calibration table is made containing only the dispersive $\delta B / \nu$ corrections that were fit to the data. Imaging the data with 


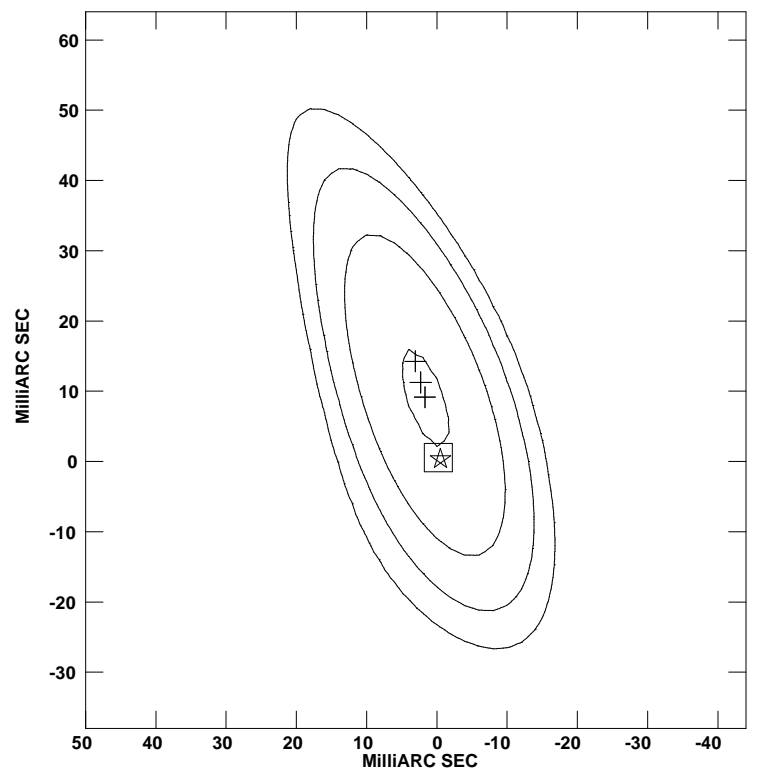

Fig. 3.- Same as figure 2 but at $1.73 \mathrm{GHz}$.

these corrections produces a new position accurate to less than a milliarcsecond. With the improved pulsar position, the fitting for $\delta B$ and imaging are repeated with all antennas. The final calibration table contains phase corrections for each antenna tabulated at time intervals of a minute or less. Figure 4 shows the $\delta B$ values for the Kitt Peakto-North Liberty baseline. This final image has much less scattered power and all frequencies appear at the same location (figure 5).

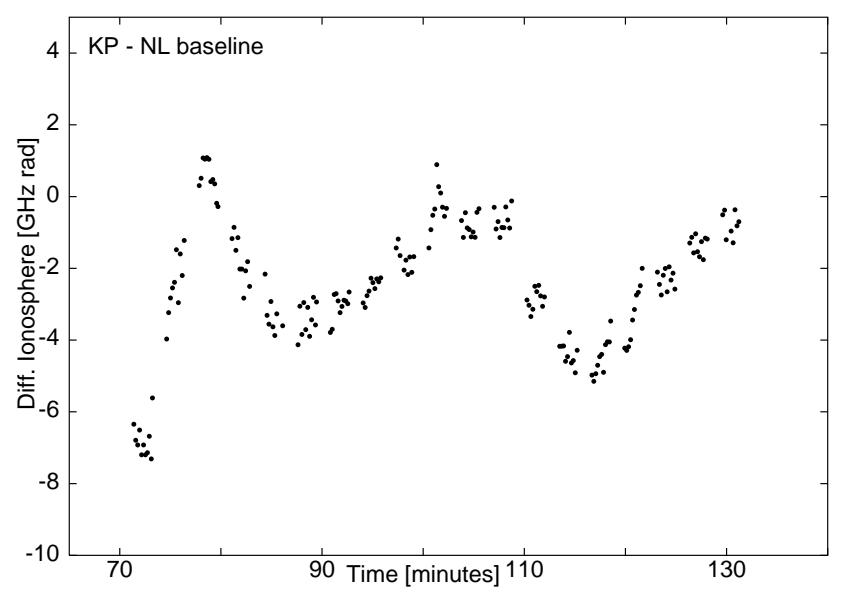

Fig. 4.- Time variation of $\delta B$ on the Kitt Peak to North Liberty baseline.
In summary, although the self-calibration process is used to derive antenna-based phases over short periods of time, only the dispersive part of these phase terms is removed, removing the ionospheric distortions. The non-dispersive part of the phases which contain information about the source position (as well as small atmospheric terms) remain in the data.

\section{Application of Ionospheric Removal to PSR B0950+08}

PSR B0950+08 has a published parallax of $7.9 \pm 0.8$ mas (Gwinn 1986). This measurement was made with a three element VLBI array consisting of Arecibo, the $140 \mathrm{ft}$. antenna at Green Bank and the $40 \mathrm{~m}$ antenna at Owens Valley. A 3-element interferometer produces a beam pattern with severe lobe ambiguity. To break the degeneracy of the solution, the proper motion was used to select a self-consistent set of lobes. Unfortunately, the published values of $\mu_{\alpha}=15 \pm 8 \mathrm{mas} / \mathrm{yr}$ and $\mu_{\delta}=31 \pm 4 \mathrm{mas} / \mathrm{yr}$ (Lyne et al. 1982) were not sufficiently accurate, causing an incorrect choice of lobes.

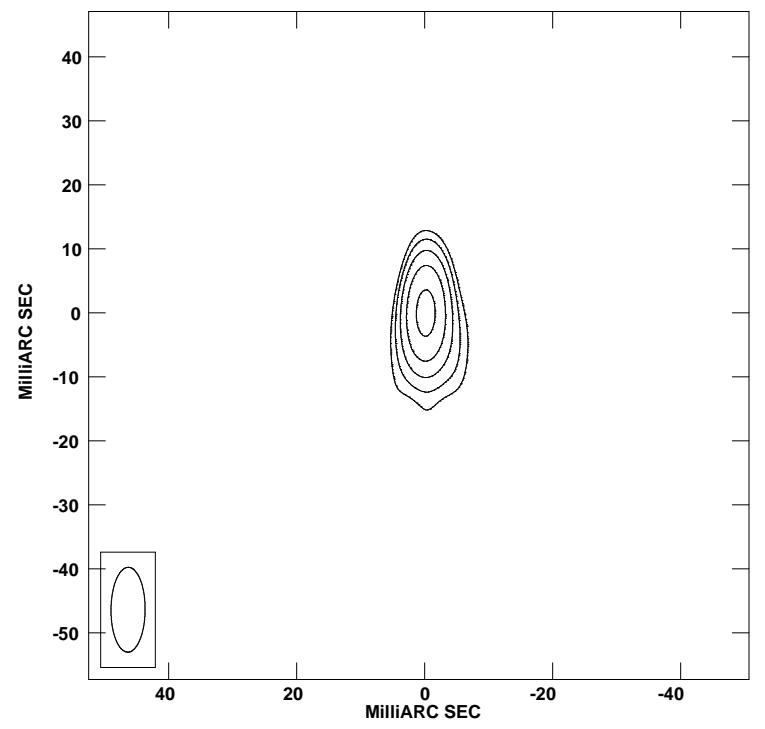

Fig. 5.- Image of the pulsar after removing the ionosphere. The same region as figure 1 is shown with the same contour levels. The highest contour is at $64 \mathrm{mJy}$

The four gated epochs of data were calibrated as described and fit with a model with five components: position of pulsar (two components), 
proper motion of pulsar (two) and parallax (one). The fit is shown in figure 6 , and the proper motion and parallax are:

$$
\begin{aligned}
\mu_{\alpha} & =-1.6 \pm 0.4 \mathrm{mas} / \mathrm{yr} \\
\mu_{\delta} & =29.5 \pm 0.5 \mathrm{mas} / \mathrm{yr} \\
\pi & =3.6 \pm 0.3 \mathrm{mas}
\end{aligned}
$$

The first three epochs of data suggest a parallax of $4 \pm 1$ mas suggesting some stability of correction even with a calibrator separation of 7 degrees. The nutation model in the correlator may leave an annual signature with an amplitude of about 40 $\mu$ as per degree of separation (C. Ma et al. 1998), about 0.1 mas in this experiment. Systematic errors such as these are not included in the error bars but are expected to be less than 0.3 mas.

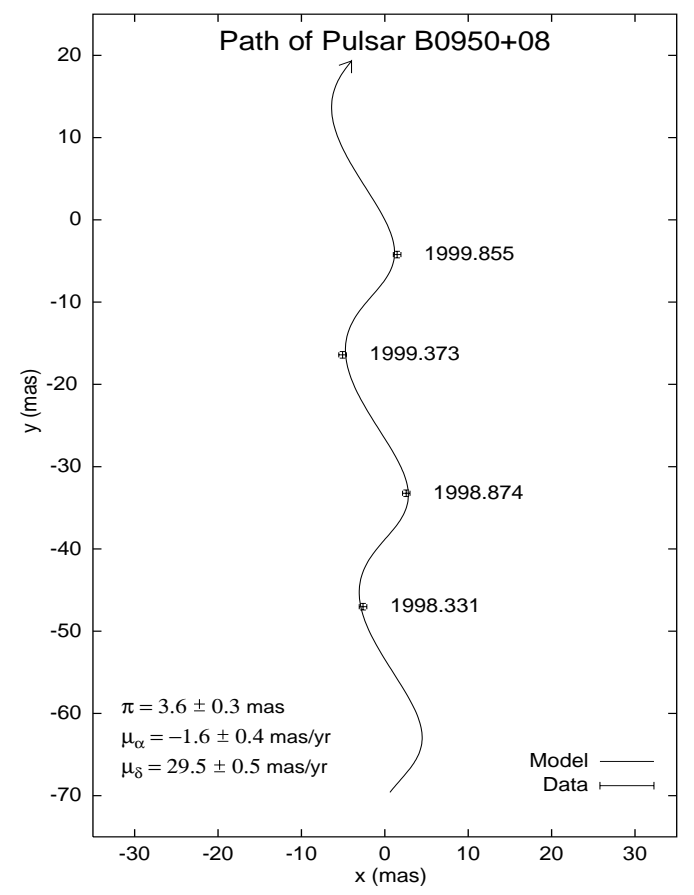

Fig. 6. - Location of pulsar at four epochs and model fit to the points.

\section{Implications}

There has been a long-standing problem of reconciling the electron density derived from the distance to PSR B0950+08 with that based on Xray data (Toscano et al. 1999). This measurement puts the pulsar more than twice as far away, changing the local electron density from $n_{e}=$ $0.023 \mathrm{~cm}^{-3}$ to $n_{e}=0.01 \mathrm{~cm}^{-3}$, a value much closer to the estimated electron density inside our local bubble, $n_{e} \approx 0.005 \mathrm{~cm}^{-3}$.

The National Radio Astronomy Observatory is a facility of the National Science Foundation operated under cooperative agreement by Associated Universities, Inc. This reasearch was funded in part by the National Science Foundation including a graduate student fellowship. Additional funding was provided by Princeton University. S.E.T. is a Sloan Research Fellow.

\section{REFERENCES}

Bhat, N. D. Ramesh, Gupta, Yashwant \& Rao, A. Pramesh 1998, ApJ, 500, 262.

Fomalont, E. B., Goss, W. M., Beasley, A. J. \& Chatterjee, S. 1999, AJ, 117, 3025.

Gwinn, C. R., Taylor, J. H., Weisberg, J. M., \& Rawley, L.A. 1986, AJ, 91, 338.

Lyne, A. G., Anderson, B. \& Salter, J. M. 1982, MNRAS, 201, 503.

Ma, C. et al. 1998, AJ, 116, 516.

Synthesis Imaging in Radio Astronomy II, Taylor, G. B., Carilli, C. L., \& Perley, R. A., 1998, ASP Conference Series, Vol. 180. 1999

M. Toscano et al. 1999, ApJL, 523, 171.

This 2-column preprint was prepared with the AAS LATEX macros v5.0. 